\title{
Influence of multiaxial cyclic plastic loading on grain boundary misorientation profile in austenitic stainless steel
}

\author{
Rima Dey, ${ }^{1}$, Soumitra Tarafder $^{2}$, and $S$ Sivaprasad ${ }^{2}$ \\ ${ }^{1}$ Academy of scientific and innovative research (AcSIR), Ghaziabad 201002, India \\ ${ }^{2}$ CSIR-National metallurgical laboratory, Jamshedpur 831007, India
}

\begin{abstract}
LN stainless was subjected to multiaxial loading employing different waveforms and load paths. The grain boundary misorientation profile so obtained post deformation was compared across loading conditions. It was found that the uniaxial and proportional conditions of loading result in more of twins, contrary to non-proportional loading conditions that resulted in substantially higher low angle grain boundaries. Also, under non-proportional loading the trapezoidal load path resulted in remarkably altered distribution of the grain boundaries compared to its contemporaries. Predominant martensitic transformation was found to be dominant mechanism of deformation for trapezoidal loading that also contributed to the altered misorientation profile.
\end{abstract}

\section{Introduction}

Based on the alterations in principal loading directions multiaxial fatigue can be sorted into proportional and non-proportional loadings. Loading histories resulting from fixed direction of cyclic loading are defined as proportional or in-phase loading. On the contrary, nonproportional loading paths result in altering directions of strains/ stress continually, thereby impacting material's macro and micro properties [1,2]. More so because of substantial cyclic hardening associated with such biaxial loadings, that principally are dependent on material as well the loading path [3-10]. For materials such as 304LN stainless steel, which is liable to deform via planar slip by virtue of its low stacking fault energy of approximately $25 \mathrm{~mJ} / \mathrm{m}^{2}$, substantial degree of additional hardening under non-proportional loading conditions relative to its proportional counterpart has been reported consistently [4,11\&12]. In addition, loading path variation is liable to immensely influence the slip system in a material, thereby leading to a wide variety of defects such as stacking faults, twins, micro bands, diverse dislocation arrangements etc., and lead to an overall high defect density across the matrix $[8,11 \& 12]$. Further, martensitic transformation has also been examined for 304LN SS under non proportional loading conditions [12]. Recording these

1 Corresponding author: rims.dey@gmail.com 
substructural features using electron microscope (eg. transmission electron microscope) is a direct way of identifying the defects, however, for such evaluations to be representative of the bulk material behaviour wherein a quantitative assessment is doable, sufficient repetition is required, which is not feasible practically. Therefore taking a cue from earlier studies, considering the fact that the substructure experiences a change under nonproportional loading which is remarkably different from its proportional counterpart, the same is anticipated to reflect in the grain boundaries, which may endow us with a likelihood of predicting state of damage in material substructurally without a detailed microstructural analysis.

\section{Experimentals}

Multiaxial strain controlled experiments were conducted using a servo-hydraulic closed loop testing system (Instron 8800) of $100 \mathrm{kN}$ axial and $1000 \mathrm{~N}-\mathrm{m}$ torsional capacity for six different waveforms as elucidated in Fig.1. All tests were carried out with tubular specimens of $1.4 \mathrm{~mm}$ wall thickness in the gauge portion and $65 \mathrm{~mm}$ gauge length which were fabricated in accordance with ASTM E 2207. An Epsilon made bi-axial extensometer of $25 \mathrm{~mm}$ gauge length with capacity of $\pm 10 \%$ axial strain and $\pm 2.5 \%$ shear strain was used for controlling strain under varied loading conditions. All tests were carried out at an equivalent von-Mises strain amplitude of $0.5 \%, 0.7 \%, 0.8 \%$ and $1 \%$ respectively. Further all values of the torsion strain and torque reported in this were converted to their corresponding von-Mises equivalent using the relation for equivalent stress $\sigma_{\text {eq }}=\sqrt{ } 3 \tau$ and equivalent strain $\varepsilon_{\mathrm{eq}}=\gamma / \sqrt{ } 3$ where $\tau$ and $\gamma$ are the respective torque and shear strain.

In order to understand the micro-mechanisms involved during multiaxial deformation, samples post failure, were cut along the gauge length at a distance uniformly pre-fixed from the fractured region. All specimens were then mechanically polished, followed by electro polishing and subjected to electron back scatter diffraction (EBSD) in scanning electron microscope at $0.4 \mu \mathrm{m}$ step size. The scanned data was processed and analyzed using TSL OIM (EDAX) software. After EBSD the same specimens were polished down to approximately $0.3 \mathrm{~mm}$ thickness with emery paper, followed by jet polishing in ethanol and perchloric acid. These specimens were then examined in CM 200 transmission electron microscope for microstructural examination at an operating voltage of $200 \mathrm{kV}$.

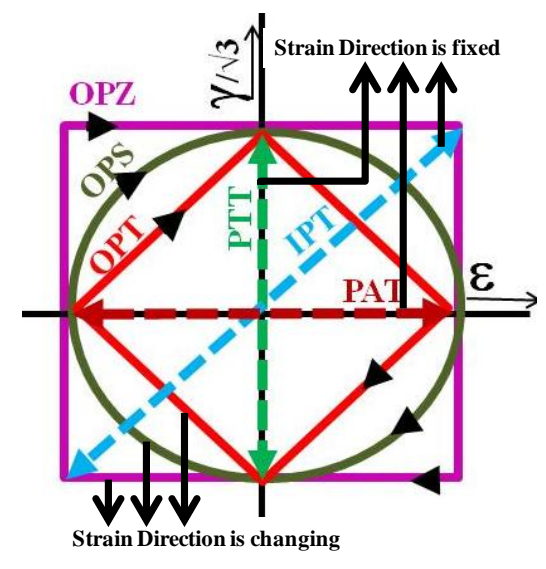

Fig. 1. Different strains path employed in this study 
A number of abbreviations are used in this paper to denote the multiaxial test parameters and electron back scatter analysis. For the sake of clarity and convenience to the readers, these abbreviations are listed in Table. 1

Table 1. List of abbreviations used

\begin{tabular}{|l|c|}
\hline PAT & Pure axial triangular \\
\hline PTT & Pure torsion triangular \\
\hline IPT & In phase triangular \\
\hline OPT & Out of phase triangular \\
\hline OPS & Out of phase sinusoidal \\
\hline OPZ & Out of phase trapezoidal \\
\hline TEM & Transmission electron microscopy \\
\hline EBSD & Electron back scatter diffraction \\
\hline LAGB & Low angle rain boundaries \\
\hline MAGB & Medium angle grain boundaries \\
\hline HAGB & High angle grain boundaries \\
\hline GBMM & Grain boundary misorientation map \\
\hline
\end{tabular}

\section{Results and discussions}

From Fig.1, for the uniaxial and proportional cases of loading which are PAT, PTT and IPT respectively, principal strain directions are fixed. For triangular waveform , as loading changes from PTT to OPT principal strain direction also changes as in Fig.1. The same is true for other out of phase conditions employing sinusoidal (OPS) and trapezoidal (OPZ) waveforms implying, increased severity in deformation conditions.

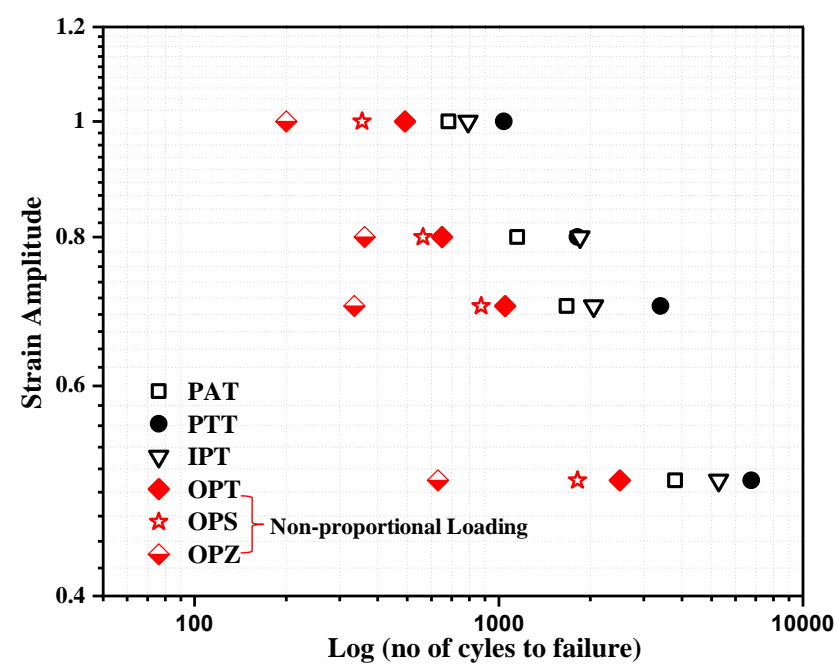

Fig. 2. Fatigue life comparison for different strain paths

Correspondingly, the fatigue life shows a substantial difference as the loading path changes. Fig. 2 compares the fatigue life for different conditions of loading where in the 
data marked in red symbolises the non-proportional loading conditions and the black colored symbols represent the uniaxial and porportional conditions of loading. It can be seen that the non-proportional loading results in least life where the order in which the life decreases with the loading path follows the sequence trianular $(\mathrm{OPT})>$ sinuoidal (OPS)> trapezoidal(OPZ). The fatigue life is supposedly a macro manifestation of micro alteration the material undergoes to accommodate fatigue deformation. Indeed grain boundary (GB) distribution plot in Fig.3[13] wherein the variation in GB perimeter per unit area for different conditions of multiaxiality are compared, shows a remarkable shift in the distribution as the loading changes from uniaxial and proportional to non-proportional.
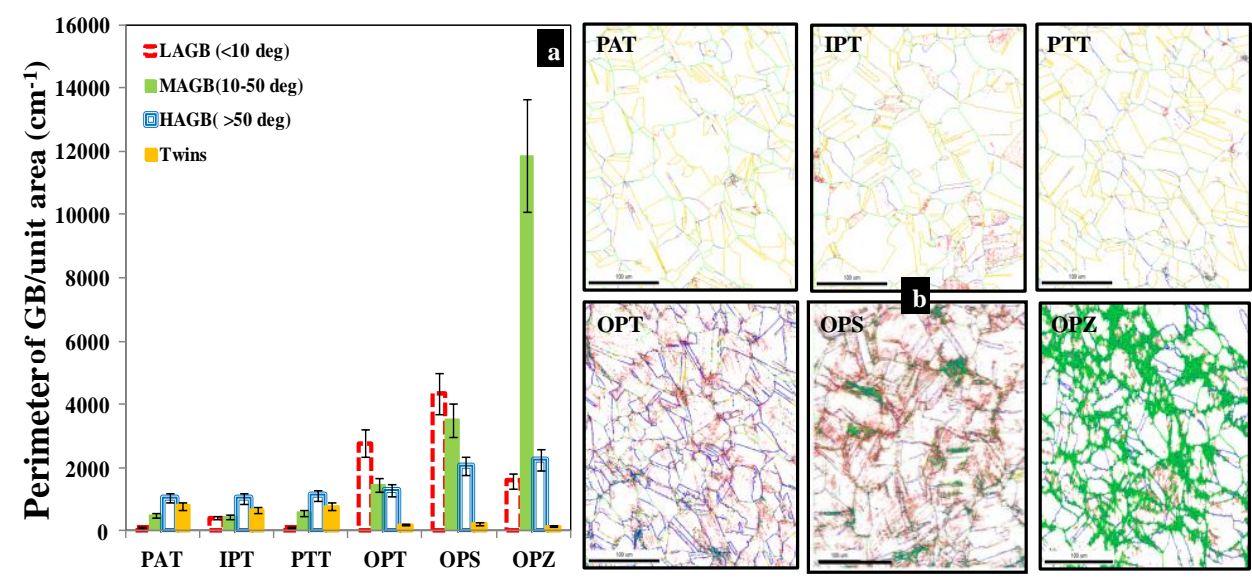

Figure.3. (a) Grain boundary character distribution plots and, (b) grain boundary misorientation maps ( with color coding, red - LAGB, green-MAGB,blue-HAGB and yellow-Twins) obtained post deformation at $1 \%$ equivalent strain

Conventionally the grain boundaries are categorised as low angle grain boundaries (LAGB with misorientation $<10^{\circ}$ ), and high angle grain boundaries (HABG with misorientation $>10^{\circ}$ ) which comprises of twins also. However this classification does not provide any infomation of the changes that happen in the misorientation range of $10^{\circ}-50^{\circ}$, if any. Considering the fact that non-proportional loading results in high concentration of defect, it is likely that this might manifest itself differently in the misorientation range of $10^{\circ}-50^{\circ}$, than other conditions of loading. Therefore, Fig. 3 shows the grain boundary profile distribution in terms of perimeter of GB per unit area for different conditions of loading obtained post defromation at equivalent strain amplitude of $1 \%$, wherein the grain boundaries have been classified as LAGB, medium angle grain boundaries (MAGB with misorientation in the range of $10^{\circ}-50^{\circ}$ ) and HAGB.

In Fig.3(a) HAGB perimeter per unit area predominates for the first three cases of loading which are PAT, IPT and PTT respectively. These are also the relatively lesser severe cases of multiaxiality, wherein the twin perimeter contributes to a larger extent to the overall HAGB fraction. Contrastinly for the non-proportional conditions of loading signified by OPT, OPS and OPZ respectively, the overall GB perimeter per unit area increases and is particularly true for the LAGB and MAGB fractions. Unlike the proportional and uniaxial loadings the propensity for twinning is significantly reduced for all non proportional loadings. Further the MAGB perimeter per unit area increases remarkably for OPZ loading compared to its contemporaries, which have a higher fraction of LAGB perimeter per unit area. This is further substantiated by the corresponding grain boundary misorientation maps (GBMM) for different conditions of multiaxiality as shown 
in Fig.3(b) which is a qualitative representation of the grain boundary misorientation plot shown in Fig.3(a). It is to noted that the same color coding as in Fig.3(a), has been used to demarcate the grain boundaries based on their misorientations wherein red, green, blue, and yellow are indicative of LAGB, MAGB, HAGB and twins respectively.

Indeed GBMM for OPT and OPS in Fig 3(b) shows presence of larger regions of LAGB marked in red, that are also distributed more homogeneously across the matrix compared to other cases. This is further substantiated by the TEM images in Fig. 4 wherein a reduction in the cell size from IPT to OPT for similar strain amplitude of $1 \%$ is visually appreciable. It is to be noted that such reduction in cell size has also been observed for OPS. For OPT and OPS activation of multiple slips systems results in increased dislocation density, which is accommodated in the cell walls resulting in more refined as well equiaxed cell structures having dense and defined cell walls that contribute to the increasing LAGB fractions. Although cell formation occured for PAT and PTT, it was limited to smaller regions as marked by the red color in the GBMM in Fig.3(b). The relatively lower fraction of LAGB is due to the inhomogeneous dislocation evolution and lack of well defined cellular arrangements as opposed to the non proportional conditions. The propensity towards cellular arrangements improved in IPT, as substantiated by the increasing LAGB fraction in Fig 3(a) and the corresponding GBMM displaying higher but localized regions marked in red. However fatigue life was not impacted severly in case of IPT and remained comparable to that of PTT and PAT. The fact that occurrence of red coloured regions are largely uniform across the matrix for OPT and OPS (Fig.3b) as opposed to localization of such regions for loading conditions where straining direction does not change, is indicative of the fact that majority of grains partake in the plasticity induced deformation due to non proportional loading conditions. However localization of red regions for PAT, IPT and PTT suggests, that only certain grains with a preferred crystallographic orientation w.r.t the applied strains accommodate the maximum deformation.
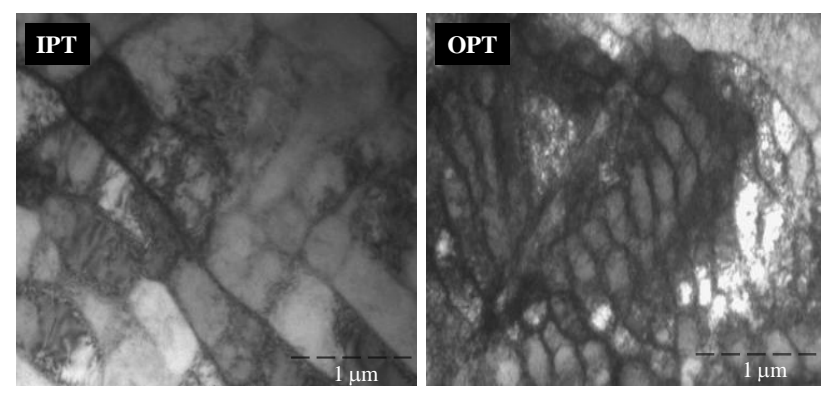

Figure.4. Cell development under IPT and OPT loading conditions

The results obtained for OPZ were strinkingly different from its contempories in terms of the misorientation data. Although the LAGB was less for OPZ the LAGB regions were scattered across the matrix as in GBMM in Fig.3(b) for OPZ, again signifying the gross participation of majority of grains in the deformation process. TEM imaging for OPZ in Fig 5(a) revealed the presence of slackly defined cells. Further their occurrence in grains was scant in comparison to OPT and OPS, which corroborate the lower LAGB in Fig.3(a) quantitatively. This also implies that proclivity to accommodate deformation through cell formation in OPZ is either pre-empted by an alternate mode of deformation. Indeed formation of large stacking faults, interacting with dislocations, coupled with intersecting shear bands surrounded by dislocation pile ups were observed in TEM for OPZ, The presence of these features are precursors of possible martensitic transformation. In fact presence of $17 \%$ martensite in the austenitic matrix post deformation for OPZ estimated through EBSD as in Fig.5(b) confirms the fact that significant phase transformation occurs 
for OPZ. The increase of MAGB possibly depicts the misoirentation across the martensitic lathe boundaries that had a mean size of approximately $0.9 \mu \mathrm{m}$. Further the distribution of the secondary phase in OPZ as shown in the phase map in Fig.5(b)was relatively homogeneous, which increases probability of martensite contributing significantly to the reduction in fatigue life observed for OPZ loading in Fig.2.
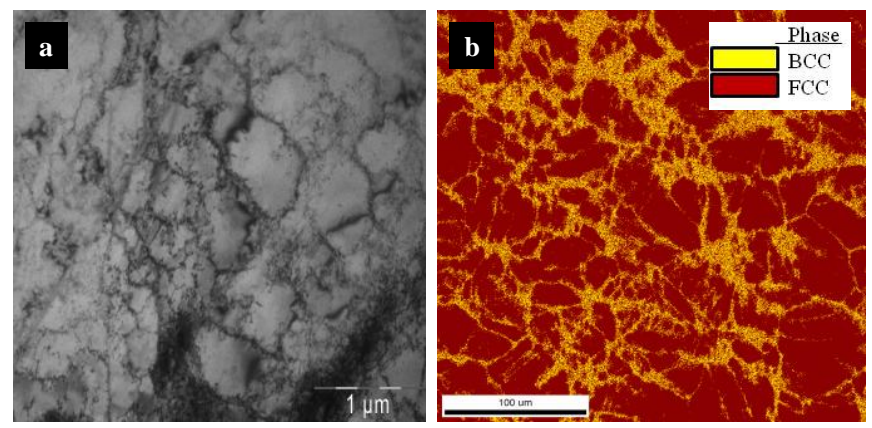

Figure.5. (a) Slack Cell development under OPZ and (b) Martensitic transformation under OPZ

\section{Conclusions}

In this study 304LN stainless was subjected to fatigue loading under both proportional and non-proportional conditions of loading, followed by electron back scatter diffraction studies post deformation wherein the results can be summarised as follows :

1. The grain boundary character is directly impacted by the type of loading. Uniaxial and proportional loading had a increased propensity towards twinning contrary to non-proportional in which tendency to accomodate deformation through twin formation decreases.

2. Cell refinement as observed for non-proportional loading employing triangular and sinusoidal waveforms result in increasing the low angle grain boundaries. However if deformation is accomodated through phase trnasformation, then it manifests itself as an increase in the medium angle grain boundaries, as was observed for trapezoidal non-proportional load path.

\section{References}

1. D.L McDowell Modeling and experiments in plasticity. Int J solids struct 37: 293-309 (2004)

2. K. Kanazawa, K. J. Miller, M. W. Brown Fatigue Eng. Mater. Struct 2, Cyclic deformationof $1 \%$ Cr-Mo-V steel under out-of-phase loads 2 :217-228 (1979)

3. E. Krempl, H. Lu. The hardening and rate-dependent behavior of fully annealed AISI type 304 stainless steel under biaxial in-phase and out-of-phase strain cycling at room temperature. ASME. J. eng. Mater Technol 106 : 376-382 (1984)

4. S.H. Doong, D. F. Socie, I. M. Robertson. Dislocation substructures and nonproportional hardening. ASME J. eng. Mater Technol, 112 : 456-464 (1990)

5. G.Z Kang, Q. Gao. Temperature-dependent cyclic deformation of SS304 stainless steel under non-proportionally multiaxial load and its constitutive modeling. Key. Eng. Mat, $274: 247-252$ (2204) 
6. E. Tanaka, S. Murakami, M.Ooka. Effects of strain path shapes on non-proportional cyclic plasticity.J. Mech. Phys. Solids 33:559-575 (1985)

7. N. Shamsaei, A. Fatemi, D. F. Socie. Multiaxial cyclic deformation and nonproportional hardening employing discriminating load paths. Int. J. Plasticity $26: 1680$ 1701 (2010)

8. T. Itoh, M. Sakane, M. Ohnami, K Ameyama. Effect of stacking fault energy on cyclic constitutive relation under non-proportional loading. J. Soc. Mat. Sci 41 :1361- 1367 (1992)

9. D.L. McDowell, D.R. Stahl, S.R. Stock, S. D. Antolovich. Biaxial path dependence of deformation substructure of type 304 stainless steel., Mettall. Trans. A 19A :1277-1293 (1988)

10. N. Shamsaei, A. Fatemi, D. F. Socie. Multiaxial fatigue evaluation using discriminating strain paths Int. J. Fatigue 33 :597-609 (2011)

11. S. Kida, T. Itoh, M. Sakane, M. Ohnami, DF. Socie . Dislocation structure and non-proportional hardening of Type 304 stainless steel, Fat. \& Fract. Eng.Mat. \& Struc. 20:1375-86 (1997)

12. DF. Socie. Multiaxial fatigue damage models. Journal of Engineering Materials and Technology. 109:293-298 (1987)

13. R.Dey, A.Das, S. Tarafder, and S. Sivaprasad. State of Grain Boundary Misorientation Due to Multiaxiality in Austenitic Stainless Steel Metall. Trans. A 50:47-51(2019) 